\title{
Competencia digital docente en el contexto de la Universidad Estatal a Distancia de Costa Rica
}

Teachers' digital competence in the context of Universidad Estatal a Distancia de Costa Rica.

\author{
Competência digital docente no contexto da Universidad Estatal a \\ Distancia de Costa Rica.
}

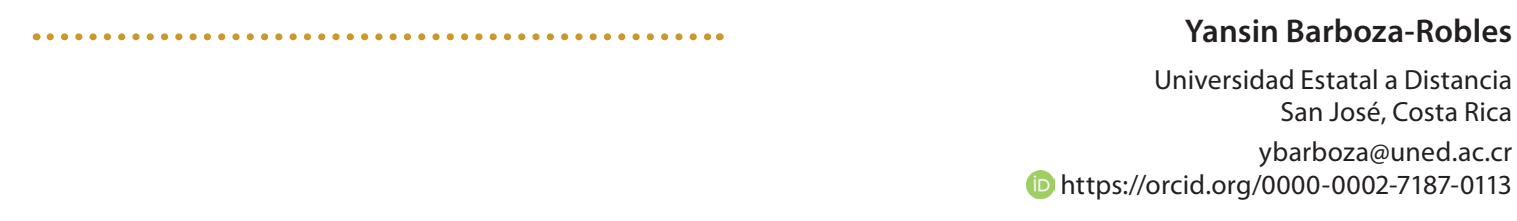

Recibido - Received - Recebido: 03/08/2020ＣＣＣｒregido - Revised - Revisado: 13/09/2020Ａceptado - Accepted - Aprovado: 25/09/2020

DOI: https://doi.org/10.22458/ie.v22i33.2954

URL: https://revistas.uned.ac.cr/index.php/innovaciones/article/view/2954

\begin{abstract}
Resumen: El artículo es producto de una investigación de enfoque cualitativo, desarrollada en la Universidad Estatal a Distancia (UNED) de Costa Rica, en el segundo semestre de 2018, con la participación de nueve personas funcionarias de la universidad, expertas en aprendizaje virtual. Su objetivo fue definir los componentes de la competencia digital docente en el contexto de la UNED, según la opinión de personas expertas en aprendizaje en línea de la institución. A partir de un grupo de enfoque, se obtuvieron 22 indicadores de logro que describen las capacidades deseables del profesorado de la UNED para desempeñarse adecuadamente en los entornos virtuales de aprendizaje, durante la fase de implementación de un curso en línea. Se recomienda utilizar la lista de indicadores para detectar necesidades de capacitación en el cuerpo docente de la institución, así como para evaluar su desempeño.
\end{abstract}

Palabras clave: competencias docentes; competencia digital; competencia digital docente; educación a distancia; educación virtual; aprendizaje en línea.

\begin{abstract}
This paper is the result of the qualitative research developed by Costa Rica's Universidad Estatal a Distancia (UNED) during 2018's second semester. It had the participation of nine of the university's experts in virtual learning. The main purpose was to define the components of teachers' digital competence considering UNED's context according to the virtual education expert's opinion. All in all, 22 achievement indicators were obtained from a focus group. Those indicators describe the UNED's teaching staff desirable skills to perform satisfactorily in virtual learning environments during
\end{abstract}


the implementation phase of an online course. The final recommendation is to use the list of indicators to detect training needs in the institution's teaching staff, as well as to evaluate their performance.

Keywords: teacher skills, digital competence, teacher's digital competence, distance learning, virtual education, online learning

Resumo: $\bigcirc$ artigo é o resultado de uma pesquisa de enfoque qualitativa, desenvolvida na Universidad Estatal a Distancia (UNED) da Costa Rica, no segundo semestre de 2018, com a participação de nove funcionários universitários, especialistas em aprendizagem virtual. Seu objetivo era definir as componentes da competência digital docente no contexto da UNED, de acordo com a opinião dos especialistas em aprendizagem on-line da instituição. A partir de um grupo focal, foram obtidos 22 indicadores de desempenho que descrevem as habilidades desejáveis dos professores da UNED para desempenhar adequadamente em ambientes virtuais de aprendizagem, durante a fase de implementação de um curso on-line. Recomenda-se que a lista de indicadores seja utilizada para identificar de necessidades de formação no corpo docente da instituição, bem como para avaliar seu desempenho.

Palavras-chaves: Competências pedagógicas; competência digital; competência digital docente; educação à distância; educação virtual; aprendizagem on-line. 


\section{INTRODUCCIÓN}

En educación, se distinguen dos modelos claramente definidos: la educación presencial y la no presencial o educación a distancia (García, 2020), la cual se ha visto enriquecida con el avance tecnológico y la proliferación de los entornos virtuales de aprendizaje (EVA), pues ha aprovechado el Internet para brindar los contenidos al estudiantado y desarrollar experiencias de aprendizaje en algunas de las asignaturas y programas que ofrece, como debía ocurrir dado que la educación a distancia requiere de la mediación tecnológica para desarrollar el proceso de enseñanza y aprendizaje.

No obstante, se debe aclarar que la educación a distancia se diferencia de la virtual o e-learning (aprendizaje virtual), aunque comparten algunas características. La educación a distancia utiliza los entornos virtuales como uno de los recursos disponibles para mediar el proceso de enseñanza y aprendizaje, junto a otros más tradicionales como los materiales didácticos escritos, el teléfono, el correo electrónico, la entrega de tareas en los centros universitarios y las tutorías presenciales. En cambio, la educación virtual es "la enseñanza a distancia que utiliza como medio de transmisión fundamentalmente Internet mediante entornos virtuales" (Rivera-Vargas, Alonso-Cano y Sancho-Gil, 2017, p. 3); es decir, la educación virtual es educación a distancia porque el estudiantado y el cuerpo docente están separados en tiempo y espacio geográfico la mayoría de las veces, pero con la salvedad de que Internet es el medio principal e insustituible para desarrollar el proceso de enseñanza y aprendizaje.

La Universidad Estatal a Distancia de Costa Rica (UNED) es una institución de educación superior pública que opera bajo el modelo a distancia y no bajo el e-learning, aunque cada vez incorpora, con mayor frecuencia, el Internet entre sus recursos. En efecto, en la actualidad dentro de su oferta académica se distinguen tres modalidades, según el grado de virtualidad (PACE, 2018):

- Regular: educación a distancia sin entornos virtuales.

- Híbrida: educación a distancia con entornos virtuales como uno más de los recursos de la asignatura, aunque un alto porcentaje de las actividades evaluadas se realiza fuera de Internet. Integra elementos del modelo tradicional a distancia como del modelo virtual.

- Virtual o e-learning: se caracteriza porque $100 \%$ de los recursos y las actividades educativas de la asignatura se desarrollan en línea.

En ese contexto de cambio metodológico en la educación a distancia, institucional e internacional, provocado por el uso de Internet y los medios digitales, es preciso valorar cuáles son las competencias que requiere el profesorado universitario para adaptarse y desempeñarse adecuadamente, a fin de facilitar el aprendizaje autónomo y autorregulado del estudiante, condición distintiva del modelo educativo a distancia.

Lo anterior alude a replantear las competencias docentes, que son el "conjunto de conocimientos, rasgos personales, actitudes y habilidades que posibilitan el desempeño de actuaciones docentes, generalmente de índole pragmática reconocible, de logro en el ámbito educativo" (Tourón, Martín, Navarro, Pradas e lñigo, 2018, p. 27).

\section{Modelos de competencias docentes}

En efecto, han existido varios modelos de competencias docentes: empezando por el popular modelo TPACK (Technological Pedagogical Content Knowledge) conformado por tres tipos de conocimientos integrados (tecnológico, pedagógico y de contenido) necesarios para el ejercicio docente (Salinas, 
De Benito y Lizana, 2014). Más adelante, en 2003, Zabalza expuso diez competencias del profesor en el marco de la calidad de la docencia universitaria, dentro de las cuales incorporó el uso de nuevas tecnologías y recursos diversos para lograr el aprendizaje y la participación del estudiantado. Luego, en 2012, Torra et al. identificaron seis competencias que deben poseer los profesores universitarios para ejercer la docencia (competencia interpersonal, metodológica, comunicativa, de planificación y gestión de la docencia, de trabajo en equipo, y competencia de innovación); en particular, dentro de la competencia metodológica, destacaron la aplicación de metodologías que consideren el uso de las TIC para contribuir a mejorar procesos de enseñanza y aprendizaje.

Por último, más recientemente, Cabero, Llorente y Morales (2018), después de analizar diversas pesquisas acerca de las competencias docentes, plantearon que el desempeño del docente virtual debe evaluarse en cuatro dimensiones: conocimiento disciplinar del contenido, conocimiento pedagógico, conocimiento tecnológico y cumplimiento de reglamentación y normas. Singularmente, el conocimiento tecnológico alude al dominio del campus virtual y de otras tecnologías; al uso pedagógico y educativo de las tecnologías; y al manejo de las herramientas tecnológicas para crear un entorno de comunicación eficaz.

De los modelos citados, se deriva que una de las competencias básicas para el profesorado es la competencia digital relativa al uso efectivo de las TIC en su práctica profesional. Así lo ha reconocido la UNESCO al proponer el Marco de competencias de los docentes en materia de TIC (ICT-CFT, tercera versión), como una herramienta para orientar la formación inicial y continua del profesorado, según los objetivos nacionales e institucionales planteados (UNESCO, 2019).

En el Marco, la UNESCO enfatiza que el cuerpo docente debe adquirir competencias para utilizar las TIC en las necesidades cotidianas de su profesión y, además, para propiciar que los mismos alumnos desarrollen estas capacidades a fin de que se transformen en miembros activos de la sociedad y en personas que aprenden durante toda la vida.

\section{Competencia digital}

La competencia digital se define como "el uso seguro y crítico de las tecnologías de la sociedad de la información (TSI) para el trabajo, el ocio y la comunicación" (Diario Oficial de la Unión Europea, 2006, p. 6), lo cual abarca el uso de dispositivos para obtener, evaluar, almacenar, producir, presentar e intercambiar información, comunicarse y participar en redes de colaboración a través de Internet.

En el contexto actual, la competencia digital forma parte de las capacidades indispensables de las personas para desenvolverse activamente como individuos, profesionales y ciudadanos, puesto que, en el siglo XXI, el conocimiento, la información, la economía y la comunicación se desarrollan en ambientes digitales.

En el campo educativo, el cuerpo docente requiere desarrollar la competencia digital por dos razones principales: la primera es que la mayoría de las instituciones utiliza Internet como parte de sus recursos didácticos; la segunda consiste en que la red es el medio que le permite al profesorado actualizar, con facilidad, los conocimientos en su campo de estudio, conformar comunidades de aprendizaje con profesionales de otras latitudes, acceder a recursos educativos abiertos y mantener una comunicación fluida con la institución educativa y el estudiantado.

Así, surgió el concepto de competencia digital docente que pretende describir los conocimientos, las habilidades y las actitudes que deben poseer los educadores para ser competentes digitalmente en el ejercicio de su profesión. 


\section{Competencia digital docente}

Por un lado, una competencia es la capacidad humana de movilizar diferentes recursos cognitivos (conocimientos, técnicas, habilidades, aptitudes) para desempeñarse capazmente en un contexto determinado, lo cual abarca realizar actividades complejas y solucionar problemas (Zabala y Arnau, 2014; Rangel, 2015). Por otro, la competencia digital docente se entiende como el conjunto de capacidades y habilidades que posibilitan la incorporación de las TIC como recurso metodológico, integrado en el proceso de enseñanza y aprendizaje, lo cual permite convertir las TIC en tecnologías para el aprendizaje y el conocimiento (TAC) con una clara aplicación didáctica (Tourón, Martín, Navarro, Pradas e Iñigo, 2018).

Por lo tanto, al hablar de competencia digital docente, interesa especificar los recursos cognitivos que debe movilizar la persona para sea catalogada como competente digital en el campo de la docencia. Entre más específica y contextualizada sea la propuesta, más útil resultará para evaluar y capacitar al profesorado.

Por ejemplo, Rangel (2015) agrupó en tres dimensiones los recursos necesarios para que el profesorado universitario integre, de manera efectiva, las TIC en su labor docente: tecnológica, informacional y pedagógica. La primera dimensión encierra los conocimientos básicos sobre el uso de las TIC, como son manejar programas ofimáticos; realizar tareas de conectividad, instalación y seguridad de la computadora; y mostrar una actitud positiva ante la actualización permanente en temas de TIC. La segunda dimensión incluye cuestiones atinentes a gestionar la información. Por último, la tercera, la dimensión pedagógica se vincula con mostrar una actitud crítica y favorable ante la posibilidad de integrar las TIC en su práctica docente; diseñar e implementar estrategias de enseñanza y aprendizaje mediadas por TIC; diseñar y evaluar materiales o recursos educativos digitales para emplearlos en su práctica docente; $y$ aprovechar las TIC para apoyar las tareas administrativo-docentes.

Por lo tanto, la propuesta de Rangel (2015) confirma la idea de que el profesorado universitario debe ser capaz de utilizar las TIC con dos finalidades: buscar, guardar, procesar y compartir información, pero también como un recurso pedagógico que le permita mejorar el proceso de enseñanza y aprendizaje.

Otro ejemplo acerca de los componentes de la competencia digital docente lo plantean Prendes, Gutiérrez y Martínez (2018), quienes sintetizaron investigaciones recientes sobre esta competencia en el contexto universitario español, como se muestra en la tabla 1. Obsérvese que la mayoría de las investigaciones identifican recursos que van desde los conocimientos básicos en el uso de la computadora, hasta la integración de las TIC para diseñar, gestionar y evaluar ambientes virtuales, formarse permanentemente, analizar el impacto social de las TIC e innovar con estas. 
TABLA 1

Competencia digital docente del profesorado universitario

\begin{tabular}{|c|c|c|}
\hline Modelo & Autor & Descripción Competencia TIC (áreas/dimensiones/ámbitos) \\
\hline $\begin{array}{l}\text { Competencia digital } \\
\text { del profesorado } \\
\text { universitario en su } \\
\text { desarrollo profesional } \\
\text { docente }\end{array}$ & Pozos (2009) & $\begin{array}{l}\text { Áreas: planificación y diseño de experiencias de aprendizaje en am- } \\
\text { bientes presenciales y virtuales; desarrollo y conducción de experien- } \\
\text { cias de aprendizaje colaborativas presenciales y en red; orientación, } \\
\text { guía y evaluación de procesos de construcción del conocimiento en } \\
\text { entornos presenciales y virtuales; gestión del crecimiento y desarrollo } \\
\text { profesional con apoyo de las TIC; investigación, desarrollo e innova- } \\
\text { ción pedagógica con/para el uso de las TIC en educación; diversidad, } \\
\text { ética y uso responsable de las TIC en desempeño profesional docente; } \\
\text { medioambiente, salud y seguridad laboral con el uso de las TIC en } \\
\text { la profesión docente. } \\
\text { Fases: básicas; profundización; generación del conocimiento. }\end{array}$ \\
\hline $\begin{array}{l}\text { Competencias TIC para la } \\
\text { docencia en la universidad } \\
\text { pública española }\end{array}$ & Prendes (2010) & $\begin{array}{l}\text { Áreas: conocimiento general del ordenador y posibilidades de las } \\
\text { TIC; conocimiento y uso de las estrategias metodológicas para el } \\
\text { trabajo en red; posibilidades y limitaciones de las TIC en el proceso } \\
\text { de enseñanza aprendizaje; elección recursos TIC para el aula; cono- } \\
\text { cimiento y uso de herramientas; publicación de material en la red; } \\
\text { uso de las TIC para diferentes tareas docentes; formación docente e } \\
\text { innovación con TIC. } \\
\text { Niveles: 1) dominio de las bases que fundamentan la acción con TIC; } \\
\text { 2) diseño, implementación y evaluación de acción con TIC; 3) análisis } \\
\text { y reflexión personal y colectiva de la acción llevada a cabo con TIC. }\end{array}$ \\
\hline $\begin{array}{l}\text { Competencia digital } \\
\text { Universidad de Lérida }\end{array}$ & $\begin{array}{c}\text { Carrera y } \\
\text { Coiduras (2012) }\end{array}$ & $\begin{array}{l}\text { Conocimiento de dispositivos, herramientas informáticas y aplicacio- } \\
\text { nes en red, y capacidad para evaluar su potencial didáctico; diseño } \\
\text { de actividades y evaluación con TIC con los estudiantes y con su } \\
\text { contexto; implementación y uso ético, legal y responsable de las TIC; } \\
\text { transformación y mejora de práctica profesional docente; tratamiento } \\
\text { y gestión eficiente de la información de la red; uso de la red para } \\
\text { trabajo colaborativo y comunicación e interacción interpersonal; } \\
\text { ayuda proporcionada a los alumnos para que se apropien de las TIC } \\
\text { y se muestren competentes en su uso. }\end{array}$ \\
\hline $\begin{array}{l}\text { CDES (Cuestionario de } \\
\text { competencias digitales } \\
\text { en educación superior) }\end{array}$ & $\begin{array}{l}\text { Mengual, Roig y } \\
\text { Blasco (2016) }\end{array}$ & $\begin{array}{l}\text { Áreas: alfabetización tecnológica; uso y acceso a la información; comu- } \\
\text { nicación y colaboración; ciudadanía digital; creatividad e innovación. }\end{array}$ \\
\hline $\begin{array}{l}\text { Competencia digital } \\
\text { docente del profesor } \\
\text { universitario }\end{array}$ & Prendes (2017) & $\begin{array}{l}\text { Áreas: técnica; información y comunicación; educativa; analítica y } \\
\text { reflexiva; y, por último, social y ética. } \\
\text { Ámbitos: docencia, investigación y gestión. }\end{array}$ \\
\hline
\end{tabular}

Fuente: Tomado de "Competencia digital: una necesidad del profesorado universitario en el siglo XXI" por M. P. Prendes, I. Gutiérrez y F. Martínez, 2018, Revista redined, 56, p. 13.

Como último ejemplo, se menciona el Modelo de Evaluación de Competencias Docentes para la Enseñanza en Línea (MECDL) de García-Cabrero et al. (2018), que agrupa las competencias en tres dimensiones: previsión del proceso de enseñanza y aprendizaje, conducción del proceso de enseñanza y aprendizaje, y valoración del impacto del proceso enseñanza y aprendizaje, como se observa en la tabla 2. 
TABLA 2

Dimensiones y competencias del MECDL

\begin{tabular}{|c|c|}
\hline Dimensión & Competencias \\
\hline $\begin{array}{l}\text { Previsión del proceso } \\
\text { de enseñanza y aprendizaje }\end{array}$ & $\begin{array}{l}\text { 1. Plantear el enfoque de la asignatura } \\
\text { 2. Planear el curso de la asignatura } \\
\text { 3. Diseñar experiencias de aprendizaje } \\
\text { 4. Usar y seleccionar TIC pertinentes } \\
\text { 5. Definir la evaluación del curso }\end{array}$ \\
\hline $\begin{array}{l}\text { Conducción del proceso } \\
\text { de enseñanza y aprendizaje }\end{array}$ & $\begin{array}{l}\text { 6. Gestionar la progresión de los aprendizajes } \\
\text { 7. Interactuar didácticamente con los alumnos } \\
\text { 8. Utilizar formas de comunicación adecuadas para apoyar el aprendizaje }\end{array}$ \\
\hline $\begin{array}{l}\text { Valoración del impacto del } \\
\text { proceso de enseñanza y } \\
\text { aprendizaje }\end{array}$ & $\begin{array}{l}\text { 9. Usar formas adecuadas para valorar el proceso de enseñanza y aprendizaje, el } \\
\text { de aprendizaje autorregulado y el colaborativo en línea }\end{array}$ \\
\hline
\end{tabular}

Fuente: Adaptado de "Las competencias docentes en entornos virtuales: un modelo para su evaluación” por B. García-Cabero, E. Luna, S. Ponce, E. J. Cisneros-Cohernour, G. Cordero, Y. Espinosa, y M. H. García, 2018, RIED: Revista lberoamericana de Educación a Distancia, 21(1), pp. 352-358.

A diferencia de los modelos anteriores, el MECDL no solo propone las nueve competencias incluidas en la tabla 2, sino que además especifica 73 acciones que debe ser capaz de ejecutar el profesorado para desempeñarse adecuadamente en la educación virtual, esta particularidad lo convierte, como indican los investigadores, en un "marco conceptual para orientar la actuación, la evaluación y la formación del profesorado virtual" (García-Cabrero et al., 2018, p. 344).

\section{Caso de la UNED respecto a la competencia digital docente}

Como en la UNED se ha incrementado el uso de los EVA en la oferta académica (PAL, 2018; UNED, 2018; Peraza-Delgado, 2020), existe un interés creciente respecto a elaborar documentos que dirijan el quehacer universitario virtual. Uno de ellos es la publicación titulada Consideraciones para el Diseño y Oferta de Asignaturas en Línea (UNED, 2017), que presenta las bases del modelo de educación a distancia de la UNED y explica las diferentes aristas que conlleva su aplicación en los entornos virtuales, tales como la mediación pedagógica, la importancia del profesor y del estudiantado, la planificación curricular y el diseño de asignaturas en línea, la evaluación de los aprendizajes, la selección de herramientas de la plataforma, así como las estrategias y técnicas que se pueden desarrollar en dichas herramientas, los recursos y los materiales didácticos, entre otras.

Respecto a los roles del profesor, con base en varios autores (Garrison y Anderson, 2005; Cabero y Román, 2006; García et al., 2007; Garrison y Vaughan, 2008, citados en UNED, 2017), se distinguen tres roles: el organizativo, el social y el pedagógico. El organizativo encierra todo lo que el docente debe desarrollar para planificar la asignatura en línea. El social implica las acciones que ejecuta el profesor para construir una comunidad de aprendizaje con las personas participantes de la asignatura, en la cual impere el respeto, la libertad de expresión, la colaboración y la construcción conjunta de conocimientos. El pedagógico atañe a las intervenciones que realiza el docente para facilitar y motivar el aprendizaje activo del estudiantado. 
Lo anterior se complementa con las competencias del profesor en el marco de la calidad según Zabalza (2003, citado en UNED, 2017), que incluye diez competencias:

1. Diseño y planificación de la docencia con sentido de proyecto formativo

2. Organización de las condiciones y del ambiente de trabajo

3. Selección de contenidos interesantes y forma de presentación

4. Materiales de apoyo a los estudiantes

5. Metodología didáctica

6. Incorporación de nuevas metodologías y recursos diversos

7. Atención personal a los estudiantes y sistemas de apoyo

8. Estrategias de coordinación con los colegas

9. Sistemas de evaluación utilizados

10. Mecanismos de revisión del proceso

Con ese antecedente, el propósito de la investigación presentada en este artículo fue establecer un modelo de la competencia digital docente en el contexto de la UNED de Costa Rica, avalado por el equipo de profesionales expertos del Programa de Aprendizaje en Línea (PAL) de la institución, con miras a construir un instrumento para evaluar la competencia en poblaciones específicas.

Para lograr el objetivo, se plantearon los siguientes objetivos específicos:

1. Determinar si existe un modelo institucional respecto a los componentes de la competencia digital docente en el contexto de la UNED, a partir de la búsqueda en investigaciones o documentos elaborados por personal de la UNED en los últimos cinco años.

2. Identificar modelos de competencias docentes que sirvan de base para construir la lista de indicadores en el contexto institucional actual.

3. Definir la lista de indicadores de logro de la competencia digital docente a través de un grupo de enfoque con funcionarios expertos en aprendizaje en línea de la UNED.

En suma, las razones que motivaron el estudio son las siguientes: (1) la cantidad de cursos y asignaturas con algún grado de virtualidad en la UNED se ha incrementado en los últimos años (en 2012, se habilitaron 1066 entornos y, en 2018, 1960, según PAL, 2018 y UNED, 2018); (2) hasta la fecha, no existía claridad sobre los componentes de la competencia digital docente en el contexto particular de la UNED; (3) los productores académicos del PAL han identificado deficiencias en las competencias docentes gracias a la asesoría que brindan a las personas tutoras (Gamboa y Mora, 2015); (4) la necesidad institucional de contar con personal docente digitalmente competente para cumplir con el compromiso institucional de emplear la metodología de la educación a distancia de una manera innovadora y flexible, lo cual significa aprovechar al máximo las TIC y recurrir a metodologías presenciales solo cuando sea necesario para cumplir los objetivos educativos y democratizadores del modelo a distancia (UNED Asamblea Universitaria Representativa, 2015); para avanzar con ese compromiso, uno de los primeros pasos era definir cuáles conocimientos y habilidades requiere el personal docente para aprovechar los medios digitales; y (5) la urgencia de contar con un instrumento de evaluación acerca de la competencia digital docente. 


\section{MATERIALES Y MÉTODOS}

La investigación fue cualitativa, pues su propósito era definir los componentes de la competencia digital docente en el contexto de la UNED, según la opinión de personas expertas en aprendizaje en línea de la institución, con miras a utilizar la información para evaluar la competencia digital docente de grupos específicos de personas tutoras.

Se optó por emplear el método cualitativo, pues favorece replantear etapas previas de la investigación para adaptar los resultados a las necesidades del contexto; también, analiza las diferentes fuentes de información para comprender el fenómeno de estudio. Lo anterior permite tener una perspectiva general, gracias a distintos puntos de mira; se basa en métodos de recolección de datos poco estructurados y preguntas abiertas, pues lo que se pretende es recabar las perspectivas de los participantes (Hernández, Fernández y Baptista, 2014).

Los datos los recolectó la investigadora, en el segundo semestre del 2018.

La metodología consistió en buscar publicaciones en el contexto de la UNED sobre la competencia digital docente, indagar sobre modelos de competencias docentes existentes a nivel internacional que sirvieran de base para alcanzar el objetivo general de la investigación y, por último, elaborar un listado de componentes de la competencia digital docente, denominados indicadores de logro, a partir de la experiencia de la investigadora en diseño, implementación y evaluación de asignaturas virtuales en la UNED y las fuentes bibliográficas citadas en la introducción.

Para definir los indicadores de logro, se consideró como fuente principal la propuesta de García-Cabrero et al. (2018), por ser reciente, específica y acorde con el modelo pedagógico de la UNED centrado en la persona que aprende; consecuentemente, los indicadores iniciales se agruparon en tres momentos: planificación, implementación y evaluación. Después, el listado se analizó con dos personas expertas en educación virtual a través de una entrevista individual semiestructurada, de manera consecutiva; posteriormente, la lista se validó con los productores académicos del PAL mediante un grupo de enfoque o entrevista grupal.

Por lo tanto, la población con la cual se realizó la investigación fue un grupo de nueve personas expertas en aprendizaje en línea de la UNED.

En cada caso, se presentó la lista de indicadores de logro a las personas participantes; se leyeron uno a uno y entre todas las personas se fueron discutiendo la pertinencia y la claridad de cada indicador, según las normas y las prácticas institucionales atinentes al aprendizaje en línea. Esta metodología derivó en eliminar ciertos indicadores, modificar otros y dividir unos cuantos, en varios indicadores, a fin de que la propuesta fuera específica y comprensible, pues se quería lograr una lista clara que se aprovechara para evaluar la competencia digital docente en la institución, así como para orientar los procesos de capacitación que se ofrecen. En la sección de resultados, se puntualiza acerca de los cambios efectuados a la lista inicial, gracias a la interacción de las personas participantes.

En síntesis, la propuesta presentada en esta publicación adapta el Modelo de Evaluación de Competencias Docentes para la Enseñanza en Línea (MECDL) de García-Cabrero et al. (2018) al contexto de la UNED, a partir del criterio experto de especialistas en el uso de las TIC dentro de la universidad, con el fin de definir los componentes de la competencia digital docente en el momento de implementación de una asignatura con componente virtual (modalidad híbrida o virtual). 


\section{RESULTADOS}

Para cumplir el primer objetivo, se buscaron investigaciones relacionadas con la competencia digital docente en el contexto de la UNED, en las revistas académicas de la UNED y en la sección de publicaciones del sitio web del PAL (https://www.uned.ac.cr/dpmd/pal/publicaciones), sin resultados positivos. De esta manera se constató que, al momento de realizar el estudio presentado en el artículo (segundo semestre del 2018), no existía otro documento o estudio más actualizado que especificara los componentes de la competencia digital docente en la institución, además de lo expuesto en la publicación Consideraciones para el Diseño y Oferta de Asignaturas en Línea (UNED, 2017), presentada en la introducción.

Continuando con el segundo objetivo específico, se indagó en la base de datos EBSCOhost acerca de publicaciones atinentes al tema de competencias docentes. Los resultados principales encontrados se mostraron en la introducción de esta publicación.

Para cumplir con el tercer objetivo específico, se realizó un grupo de enfoque, en el cual las personas participantes recomendaron centrar el estudio en el momento de implementación de una asignatura virtual, pues la mayoría del profesorado universitario participa en esa fase de un curso virtual. Al inicio, en esa fase, la investigadora consignó 13 indicadores de logro para describir las capacidades que debe tener el profesorado universitario para ser catalogado como competente digital, con base en su experiencia profesional y la bibliografía consultada.

Gracias a la interacción del grupo, el número aumentó de 13 a 22 indicadores de logro. Los cambios más significativos fueron los siguientes: incluir el significado de normas de netiqueta y otros conceptos, a fin de que el modelo fuera comprensible para aquellas personas que desconocían los tecnicismos; definir un indicador de logro para cada formato en el cual la persona tutora debe ser capaz de brindar retroalimentación; especificar que la persona tutora debe estar capacitada para brindar retroalimentación tanto de manera grupal como individual a través de los diferentes formatos; detallar los sistemas de citación más utilizados en la UNED; señalar cuáles acciones involucra "utiliza correctamente el foro de dudas" según la normativa institucional; diferenciar entre el uso técnico y pedagógico de las herramientas de la plataforma; $y$ agregar otros indicadores con base en las dificultades identificadas en las asesorías para la planificación, el desarrollo y la mediación de los EVA y en las jornadas de actualización desarrolladas por el PAL.

Las jornadas de actualización son "espacios dirigidos primordialmente a encargados de cátedras, programa y docentes involucrados en procesos de virtualización, con el propósito de dar a conocer y promover el uso de las diferentes herramientas que ofrece la plataforma LMS institucional" (PAL, 2018, p. 2). Por ejemplo, en 2018, se ofrecieron 20 jornadas de actualización a 379 personas en total sobre los siguientes temas: uso de Turnitin, juegos de Moodle, cuaderno de calificaciones, herramienta Big Blue Button, buenas prácticas y uso de dispositivos móviles en la educación a distancia (PAL, 2018). Por lo tanto, gracias a estas experiencias, las personas participantes contaban con insumos suficientes para valorar los indicadores iniciales y modificarlos de acuerdo con las particularidades de la UNED.

De esta forma, se obtuvo la lista de 22 indicadores presentada en la tabla 3, los cuales describen las capacidades que debe tener el profesorado de la UNED, en el momento de implementación de una asignatura o curso virtual, según la opinión de las personas participantes. Para cada indicador, se establecieron cinco niveles de dominio posibles con la siguiente representación: 0 , dominio nulo; 1, bajo; 2, medio; 3 , avanzado; $y$ 4, experto 
TABLA 3

Indicadores de logro de la competencia digital docente en el momento de implementación de una asignatura o curso virtual

Indicador

Nivel de dominio

\begin{tabular}{l|l|l|l|l|}
\hline 0 & 1 & 2 & 3 & 4
\end{tabular}

1. Conoce y aplica las normas de netiqueta (buenas maneras de comportamiento en los entornos virtuales).

2. Domina los programas tecnológicos de su área disciplinar.

3. Brinda retroalimentación de forma personalizada y grupal a través de texto (se define retroalimentación como la comunicación que establece el docente durante el proceso de enseñanza y aprendizaje, con la intención de dar información inmediata y oportuna al alumno acerca de la calidad de su desempeño para que realice las correcciones necesarias con el objeto de mejorar su aprendizaje).

4. Brinda retroalimentación de forma personalizada y grupal a través de audio.

5. Brinda retroalimentación de forma personalizada y grupal a través de imágenes fijas.

6. Brinda retroalimentación de forma personalizada y grupal a través de video.

7. Crea recursos complementarios en diferentes formatos para aclarar dudas conforme avanza la asignatura, de acuerdo con las características, metas y comentarios del estudiantado.

8. Busca y comparte recursos complementarios en diferentes formatos para aclarar dudas conforme avanza la asignatura, de acuerdo con las características, metas y comentarios del estudiantado.

9. Utiliza sitios de almacenamiento en la nube y herramientas para la creación de documentos de forma colaborativa.

10. Conoce y aplica mecanismos para respetar las leyes de propiedad intelectual e identificar el plagio.

11. Utiliza un sistema de citación y referencia de forma consistente en sus producciones (APA, Chicago, Vancouver, etc.).

12. Usa herramientas dentro y fuera de la plataforma para la realización de sesiones sincrónicas (estas ocurren cuando el emisor y el receptor del mensaje interactúan al mismo tiempo, por ejemplo, en una llamada telefónica).

13. Establece comunicación asincrónica a través de las herramientas de la web 2.0 (la comunicación asincrónica pasa cuando el emisor y el receptor se comunican en tiempos diferentes, como sucede en el correo electrónico o en un foro).

14. Responde a las consultas planteadas en el foro de dudas y el correo de la plataforma de forma oportuna y pertinente.

15. Maneja adecuadamente las dudas planteadas; por ejemplo: refiere a los estudiantes cuando plantean una pregunta que ya ha sido respondida en un hilo de discusión anterior, refiere al medio de comunicación adecuado cuando el mensaje no responde al propósito del foro de dudas, etc.

16. Asesora al estudiantado sobre el uso de las tecnologías y resuelve dudas básicas sobre errores y fallas de la tecnología.

17. Conoce y utiliza las herramientas de seguimiento de la plataforma para identificar estudiantes menos dispuestos o en riesgo de rezago.

18. Motiva la participación de los estudiantes, de forma personalizada y grupal, por medio de las herramientas de la plataforma (foro, correo electrónico).

19. Usa el cuaderno de calificaciones de la plataforma para brindar las calificaciones y la retroalimentación respectiva.

20. Devuelve archivos adjuntos con comentarios, indicaciones y observaciones a través de las diferentes herramientas de la plataforma.

21. Conoce y hace un manejo técnico adecuado de las herramientas de la plataforma en las actividades de aprendizaje.

22. Maneja apropiadamente las herramientas de la plataforma en las actividades de aprendizaje, desde la perspectiva pedagógica. 


\section{DISCUSIÓN}

La lista de la tabla 3 evidencia la importancia que tiene la calidad de la comunicación que llevan a cabo las personas tutoras de la UNED en los EVA, pues no solo interesa que se comuniquen, sino que lo hagan respetuosamente, aplicando las normas de netiqueta y en diferentes formatos.

Lo mismo sucede con la retroalimentación brindada al estudiantado en los entornos virtuales de aprendizaje. La retroalimentación es la comunicación que utiliza el docente durante el proceso de enseñanza aprendizaje para informar oportunamente al estudiantado sobre la calidad de su desempeño y cómo mejorarlo; por lo tanto, se dice que es "la columna vertebral en el proceso de construcción del conocimiento" (Alvarado, 2014, p. 61). Con la retroalimentación constante, clara y oportuna, el estudiantado es capaz de autorregular su aprendizaje, lo cual es indispensable en los sistemas de educación a distancia.

Asimismo, la capacidad del profesorado de brindar retroalimentación tanto de forma individual como grupal permite maximizar el esfuerzo que conlleva brindar retroalimentación, especialmente, cuando se elabora en formato de audio o video. Aunque se debe aclarar que la plataforma Moodle utilizada en la UNED facilita brindar retroalimentación en audio o video desde la misma ventana donde se califica la tarea, sin necesidad de utilizar softwares de grabación externos. En la figura 1, se observan las posibilidades de la ventana de calificación de la plataforma.

El profesorado universitario debe aumentar el uso de estas herramientas, pues se sabe que aportan proximidad y detalle a la retroalimentación, gracias al efecto positivo que tiene en el estudiantado escuchar la voz de la persona docente. En efecto, hay evidencia que permite afirmar que el alumnado encuentra más útil, detallada y fácil de entender la retroalimentación a través de audio y video, en comparación con la retroalimentación escrita (García-Jiménez, 2015).

Figura 1. Captura de pantalla de la ventana de calificación de la plataforma Moodle

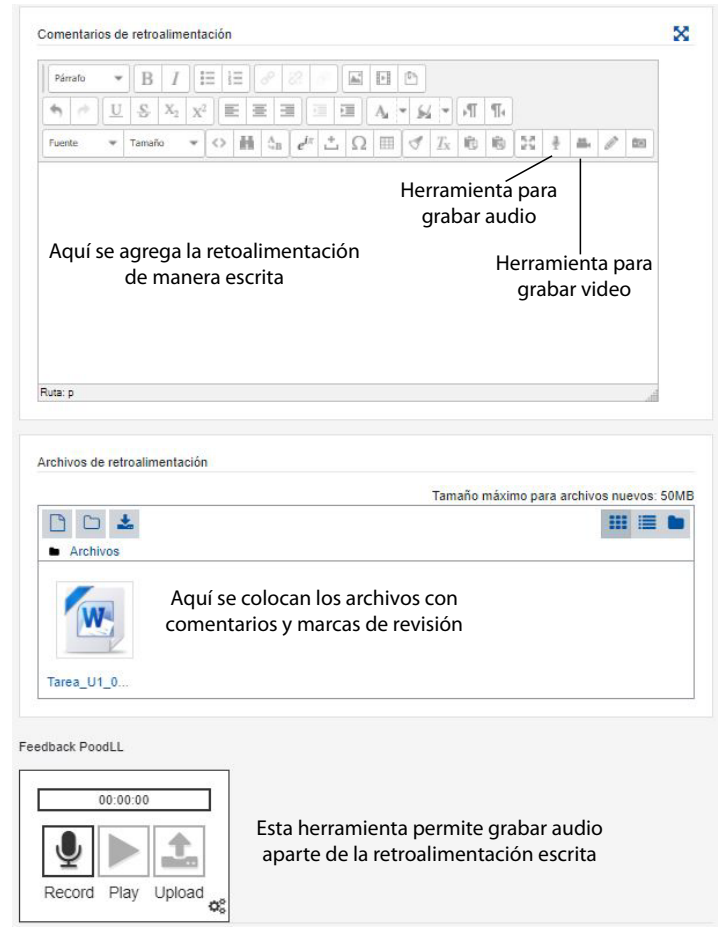

Fuente: Elaboración propia (2020). 
Para que la retroalimentación cumpla las características de ser constante, clara, oportuna, suficiente y pertinente, se requiere compromiso y disciplina por parte de la persona tutora, pues debe ingresar a diario al EVA y brindar la retroalimentación en el formato que mejor se ajuste a las necesidades del estudiantado (Alvarado, 2014).

En todo caso, es una realidad que la mayoría de las comunicaciones e intervenciones en los EVA se ejecuta de manera escrita; por lo tanto, es deseable que el profesorado se preocupe por redactar correctamente, tanto respecto al orden de las ideas expuestas como a la sintaxis, la ortografía y la puntuación, pues su forma de comunicación representa un modelo para el estudiantado. Es decir, el profesorado debe cuidar no solo el contenido de sus comunicaciones, sino también la forma de estas.

El hecho de que se incluya la capacidad de elaborar recursos en diferentes formatos entre los indicadores de logro de la competencia digital docente, constata el interés institucional de adaptar el formato de los mensajes a las necesidades del estudiantado, lo cual es coherente con la máxima de educación inclusiva que promulga la UNED.

Para favorecer la educación inclusiva, el 21 de febrero del 2019, el Consejo Universitario de la UNED aprobó la Política para la Implementación del Diseño Universal para el Aprendizaje (DUA), en la cual se establece el DUA como base de la actividad docente (UNED Consejo Universitario, 2019). El paradigma se compone de tres principios y uno de ellos es proporcionar múltiples medios para representar la información. En consecuencia, el cuerpo docente de la UNED debe desarrollar capacidades para elaborar recursos en diferentes formatos, a fin de responder a la política mencionada que busca beneficiar el aprendizaje de la diversidad del alumnado.

En esa misma línea, la habilidad de crear recursos complementarios en diferentes formatos para aclarar dudas conforme avanza la asignatura, de acuerdo con las características, las metas y los comentarios del estudiantado, se esperaría que influya en el logro de los objetivos de aprendizaje por parte de la población estudiantil particular que se trate, pues se corregirán los vacíos que surjan sobre la marcha del curso con componente virtual. Además, los recursos complementarios podrían utilizarse para mejorar la oferta siguiente de la asignatura y servir de base para producir materiales específicos con el apoyo de la Dirección de Producción de Materiales Didácticos (DPMD) de la UNED.

Por otro lado, el hecho de que el profesorado universitario utilice sitios de almacenamiento en la nube le permitirá evitar dificultades relacionadas con la capacidad máxima de almacenamiento de la plataforma virtual. A la vez, mediante las herramientas para la creación de documentos de forma colaborativa, se puede potenciar el trabajo colaborativo en los cursos virtuales.

Con respecto a los indicadores 10 y 11, es básico que el profesorado universitario utilice un sistema de citación de manera consistente en sus producciones e intervenciones en el aula virtual, con el objetivo de modelar y potenciar la capacidad en el alumnado, lo cual responde a una correcta ética profesional y académica. Lo mismo sucede con la aplicación de mecanismos para respetar las leyes de propiedad intelectual e identificar el plagio. En efecto, a nivel internacional existe una preocupación creciente respecto al aumento del plagio académico y "la necesidad de una formación ética como aspecto inherente a los planes de estudio y a los contextos de aprendizaje universitarios" (Muñoz-Cantero, Rebollo-Quintela, Mosteiro-García y Ocampo-Gómez, 2019, p. 12).

Con el objetivo de prevenir el plagio, se recomienda incluir en las orientaciones académicas o el programa de la asignatura qué es el plagio y las sanciones a las que se expone el estudiantado al cometerlo, especificar el sistema de referenciación que se utilizará en la asignatura, incluir el uso adecuado de referencias dentro de los criterios de evaluación de las producciones estudiantiles, usar software para la detección de coincidencias, ofrecer informes de coincidencias en la retroalimentación de las tareas y visibilizar, en la plataforma, la referencia completa de cada recurso utilizado que no sea de la autoría de la persona docente encargada de la asignatura (Turnitin, s. f.). 
Si bien las personas productoras académicas del PAL enfatizaron en que recomiendan utilizar las herramientas de comunicación sincrónica de la plataforma, en el indicador 12 se decidió mantener la posibilidad de que la herramienta empleada esté fuera de la plataforma, a fin de que el profesorado cuente con otras opciones ante fallas técnicas con la herramienta interna. Sin embargo, se aclara que el profesorado debe evidenciar las actividades y los resultados de aprendizaje en la plataforma institucional, dado que es el medio de interacción oficial.

Aunque en los EVA se prefiere la comunicación asincrónica para no limitar a un horario específico la participación del estudiantado, el profesorado universitario debe saber cómo realizar sesiones sincrónicas por la cercanía y la confianza que estas permiten desarrollar. Dicho conocimiento comprende tanto el uso técnico de las herramientas como el manejo pedagógico del grupo en este tipo de encuentros en tiempo real. Además, se aconseja grabar las sesiones sincrónicas para que las personas a quienes se les dificultó la participación puedan reproducir la sesión posteriormente.

En particular, las herramientas de comunicación asincrónica se catalogan como Tecnologías para el Empoderamiento y la Participación (TEP), pues posibilitan la producción libre de ideas respecto a un tema, lo cual origina puentes entre el conocimiento individual y el colectivo, así como el empoderamiento del estudiantado y el desarrollo de capacidades de producción (Villarreal-Villa, García-Guliany, HernándezPalma y Steffens-Sanabria, 2019). Esto sucede con los foros virtuales que permiten discutir sobre un tema de interés a fin de desarrollar el pensamiento crítico y generar, de manera colectiva, conocimiento fundamentado (UNED, 2017).

Con los indicadores 14 y 15, se consideró relevante que la persona tutora sepa manejar adecuadamente las dudas planteadas y se especificaron algunas de las estrategias recomendadas para hacerlo, como referir a las personas cuando plantean una pregunta que ya ha sido respondida en un hilo de discusión anterior y referir al medio de comunicación adecuado cuando el mensaje no concuerda con el propósito del foro de dudas. Abrir espacios para la atención permanente de las dudas y las consultas del estudiantado, así como el adecuado seguimiento de los mensajes expuestos en estos espacios (tanto en forma como en tiempo de respuesta), repercutirá de manera considerable en la motivación y el desempeño del alumnado en las experiencias de aprendizaje planificadas en la asignatura (UNED, 2017). De hecho, la atención oportuna de las dudas e inquietudes presentadas forma parte de los criterios de calidad por considerar en la oferta de asignaturas en línea (Salas y Umaña, 2017).

Respecto al indicador 16, sobre asesorar al estudiantado en el uso de las tecnologías y fallas tecnológicas, si bien el PAL y la Dirección de Tecnología de Información y Comunicaciones (DTIC) son las instancias encargadas de solucionar los problemas técnicos de la plataforma virtual de aprendizaje institucional, se considera que el profesorado de la UNED debe contar con la pericia para asesorar al estudiantado sobre problemas básicos, con el objetivo de solucionar las dificultades de manera oportuna y aprovechar la asesoría de las personas especialistas en tecnología solo en casos necesarios.

Por otro lado, para disminuir la deserción escolar en el modelo a distancia, es fundamental que el profesorado conozca y pueda emplear las herramientas de seguimiento de la plataforma con la finalidad de identificar personas en riesgo de abandonar el curso oportunamente, ofrecerles ayuda y aumentar el acompañamiento, cuando lo requieran; esto se evidencia en los indicadores 17 y 18 de la tabla 3. García (2019) identificó el escaso seguimiento y la supervisión docente como una de las causas del abandono escolar en los sistemas de educación a distancia atribuibles al cuerpo docente.

Finalmente, se incluyó el uso del cuaderno de calificaciones dentro de la lista de indicadores, pues este permite tener un registro ordenado y actualizado de las calificaciones, visible para el alumnado y el profesorado, lo cual garantiza la transparencia del proceso de evaluación. Además, el hecho de que el profesorado devuelva archivos con observaciones específicas le ayudará al estudiantado a identificar sus falencias y corregirlas. Cabe destacar la relevancia de la retroalimentación detallada como parte de la 
evaluación formativa, como se explicó anteriormente; asimismo, en el proceso evaluativo es básico que la persona evaluadora cumpla con los plazos estipulados por la institución para devolver la calificación y la retroalimentación correspondiente al estudiantado.

Como se indicó antes, es igual de importante que el profesorado utilice adecuadamente las herramientas de la plataforma en las actividades de aprendizaje, desde la parte técnica como desde la parte pedagógica. Por ejemplo, en el caso de las herramientas para las sesiones sincrónicas, no es suficiente con que el profesorado sea capaz de configurar la herramienta; también debe manejar este tipo de experiencias de aprendizaje como un todo, lo cual concierne acciones antes, durante y después de la sesión. Antes de la sesión, se aconseja proponer diferentes horarios y habilitar herramientas dentro de la plataforma para que el estudiantado confirme su participación; además, debe definir claramente los objetivos y la agenda de la reunión, comunicar los objetivos, la agenda y los materiales que requerirá el estudiantado durante la sesión, así como planificar los recursos necesarios. Durante la sesión, debe comenzar la reunión antes de su inicio oficial, dar la bienvenida al estudiantado, comunicar la agenda y las reglas de participación, así como aprovechar todas las posibilidades de interacción y comunicación de la herramienta (chat, compartir pantalla, pizarra, hacer grupos, etcétera); a la vez, debe respetar el tiempo definido para la reunión, ser conciso en sus intervenciones, propiciar la participación del estudiantado y realizar la grabación de la videoconferencia para que pueda ser accedida posteriormente. Después de la reunión, debe revisar la grabación, compartir los materiales utilizados y comunicarle al grupo cuando esté disponible la grabación (PACE y PAL, 2018; Gómez y Prado, 2020).

Por lo tanto, como se deduce del ejemplo anterior, la persona docente facilitadora de asignaturas con componente virtual debe ser capaz de aprovechar las herramientas de la plataforma en todas sus dimensiones; asimismo, los esfuerzos de capacitación y actualización docente deben orientarse en ambas vías (desde lo técnico y desde lo pedagógico), a fin de suplir las necesidades de aprendizaje del estudiantado con las herramientas disponibles en la plataforma virtual.

\section{CONCLUSIONES}

En la introducción se evidenció un cambio metodológico en la educación a distancia derivado del uso de los entornos virtuales y otras herramientas digitales. Aunque el inicio del modelo de educación a distancia no es reciente, pues su primera generación (la enseñanza por correspondencia) data de finales del siglo XIX y principios del XX (García, 1999), sin duda es un modelo vivo, en constante modificación, dado que necesita de la mediación tecnológica para suplir la separación física y temporal entre el estudiantado y el profesorado que la caracteriza. Por lo tanto, al variar la tecnología usada como medio de comunicación entre las partes, deben modificarse también las capacidades docentes para desenvolverse adecuadamente en el contexto actual.

Asimismo, se mostró que la oferta de asignaturas híbridas y virtuales en la UNED viene en aumento, igual que la cantidad y la variedad de herramientas tecnológicas; por ende, cada año se requiere personal calificado para mediar las asignaturas con componente virtual.

Al examinar publicaciones y documentos recientes elaborados por personal de la institución, se logró determinar que existía un vacío respecto a cuáles son específicamente los componentes de la competencia digital docente en el contexto de la UNED; es decir, no había un modelo institucional.

En la investigación, se buscaron referentes teóricos tocantes a modelos de competencias docentes que sirvieran de base para construir la lista de indicadores de la competencia digital docente en el contexto de la UNED. En la introducción, se mostraron los modelos analizados, incluido el MECDL de García-Cabrero et al. (2018), en el cual se fundamentó mayoritariamente la propuesta inicial que luego mejoraron las personas participantes. 
En ese contexto, el trabajo es relevante, pues permitió definir los componentes de la competencia digital docente durante la implementación de un curso virtual, según la opinión de las personas expertas en aprendizaje en línea de la UNED, a partir del MECDL de García-Cabrero et al. (2018). Por lo tanto, Ilena un vacío respecto a un marco de referencia contextualizado de la competencia digital docente, que impulse la evaluación y la formación del profesorado, como lo mencionan Castro-Granados y Artavia-Díaz (2020).

La lista de componentes obtenida incluye 22 indicadores de logro atinentes a comunicación, retroalimentación, dominio de programas tecnológicos del área disciplinar, búsqueda y creación de recursos complementarios, creación de documentos compartidos, respeto a las leyes de propiedad intelectual, realización de sesiones sincrónicas, atención de dudas, seguimiento de la participación del estudiantado, uso del cuaderno de calificaciones, así como manejo técnico y pedagógico de las herramientas de la plataforma.

Los aspectos anteriores son básicos para asegurar la motivación, la participación y el aprendizaje autónomo y autorregulado del estudiantado, así como para potenciar la construcción colaborativa de aprendizajes, gracias a las posibilidades de interacción que ofrecen las TIC. Si el profesorado universitario desarrolla las capacidades descritas en los indicadores, sin duda, repercutirá en la permanencia del alumnado en el sistema de educación a distancia y la consecuente obtención de su título universitario, lo cual resultará en el éxito del cuerpo docente y la propia institución educativa (García, 2019).

Se sugiere utilizar la lista de indicadores de logro obtenida en esta investigación para evaluar las capacidades del profesorado de la UNED en el momento de implementación de una asignatura con componente virtual, a fin de identificar necesidades de capacitación que ayuden a solventar las áreas débiles. Asimismo, se aconseja aprovechar las recomendaciones expuestas en la discusión, para orientar, evaluar y autoevaluar el desempeño docente en los EVA.

Se recomienda desarrollar una investigación similar centrada en definir las habilidades específicas requeridas por las personas docentes para desempeñarse, de manera adecuada, antes y después de la implementación de un curso virtual, con la participación del personal especializado en educación virtual de la UNED.

\section{REFERENCIAS}

Alvarado, M. (2014). Retroalimentación en educación en línea: una estrategia para la construcción del conocimiento. RIED. Revista Iberoamericana de Educación a Distancia, 17(2), 59-73. Recuperado de http://revistas.uned.es/index.php/ried/article/view/12678/11873

Cabero, J., Llorente, M. del C. y Morales, J. A. (2018). Evaluación del desempeño docente en la formación virtual: ideas para la configuración de un modelo. Revista lberoamericana de Educación a Distancia, 21(1), 261-279. Recuperado de https://doi.org/10.5944/ried.21.1.17206

Castro-Granados, A., y Artavia-Díaz, K.Y. (2020). Competencias digitales docentes: un acercamiento inicial. Revista Electrónica Calidad En La Educación Superior, 11(1), 47-80. https://doi.org/10.22458/ caes.v11i1.2932

Diario Oficial de la Unión Europea, L394 (2006). Recomendación del Parlamento Europeo y del Consejo de 18 de diciembre de 2006 sobre las Competencias Clave para el Aprendizaje Permanente. Recuperado de https://eur-lex.europa.eu/LexUriServ/LexUriServ.do?uri=OJ:L:2006:394:0010:0018:ES:PDF

Gamboa, Y. y Mora Vicarioli, F. (2015). Análisis sobre las competencias mediacionales que posee el tutor virtual de la UNED de Costa Rica. Innovaciones educativas, 17(22), 27-40. https://doi.org/10.22458/ ie.v17i22.1098

García, L. (1999). Historia de la Educación a Distancia. RIED. Revista Iberoamericana de Educación a Distancia, 2(1), 8-27. Recuperado de http://revistas.uned.es/index.php/ried/article/view/2084/1959 
García, L. (2019). El problema del abandono en estudios a distancia. Respuestas desde el Diálogo Didáctico Mediado. RIED. Revista Iberoamericana de Educación a Distancia, 22(1), 245-270. https:// doi.org/10.5944/ried.22.1.22433

García, L. (2020). Bosque semántico: ¿educación/enseñanza/aprendizaje a distancia, virtual, en línea, digital, eLearning...? RIED. Revista Iberoamericana de Educación a Distancia, 23(1), 09-28. https:// doi.org/10.5944/ried.23.1.25495

García-Cabrero, B., Luna, E., Ponce, S., Cisneros-Cohernour, E. J., Cordero, G., Espinosa, Y. y García, M. H. (2018). Las competencias docentes en entornos virtuales: un modelo para su evaluación. RIED: Revista lberoamericana de Educación a Distancia, 21(1), 343-365. Recuperado de http://revistas.uned. es/index.php/ried/article/view/18816

García-Jiménez, E. (2015). La evaluación del aprendizaje: de la retroalimentación a la autorregulación. El papel de las tecnologías. RELIEVE - Revista Electrónica de Investigación y Evaluación Educativa, 21(2), 1-24. https://doi.org/10.7203/relieve.21.2.7546

Gómez, S. y Prado, J. E. (2020). Herramientas de videocomunicación para la gestión y la docencia. Centro de Capacitación en Educación a Distancia. Recuperado de https://www.uned.ac.cr/academica/images/ ceced/Herramientas_de_videocomunicacio\%CC\%81n_para_la_gestio\%CC\%81n_y_la_docencia.pdf

Hernández, R., Fernández, C. y Baptista, P. (2014). Metodología de la investigación (6.a ed.). México: Editorial Mc Graw Hill.

Muñoz-Cantero, J-M., Rebollo-Quintela, N., Mosteiro-García, J., y Ocampo-Gómez, C-I. (2019). Validación del cuestionario de atribuciones para la detección de coincidencias en trabajos académicos. RELIEVE - Revista Electrónica de Investigación y Evaluación Educativa, 25(1), 1-16. https://doi.org/10.7203/ relieve.25.1.13599

PACE (Programa de Apoyo Curricular y Evaluación de los Aprendizajes). (2018). Guía para el diseño y rediseño curricular de asignaturas. Recuperado de https://www.uned.ac.cr/academica/images/PACE/ docs/dise\%C3\%B1o_asignatura/Gu\%C3\%ADa_para_el_dise\%C3\%B1o_y_redise\%C3\%B1o_curricular_de_asignaturas_2019.pdf

PACE y PAL (Programa de Aprendizaje en Línea). (2018). Consideraciones para la selección de estrategias y técnicas que pueden utilizarse en las herramientas de la plataforma virtual de la UNED. Recuperado de https://www.uned.ac.cr/dpmd/pal/images/documentos/Profesores/consideraciones-seleccionestrategias.pdf

PAL (2018). Informe de labores anual Programa de Aprendizaje en Línea. [Documento sin publicar].

Peraza-Delgado, M. (2020). Análisis del procedimiento actual para la creación y gestión de asignaturas con componente virtual de la UNED de Costa Rica y propuesta de mejoramiento. Revista Electrónica Calidad En La Educación Superior, 11(1), 25-46. https://doi.org/10.22458/caes.v11i1.2931

Prendes, M. P., Gutiérrez, I. y Martínez, F. (2018). Competencia digital: una necesidad del profesorado universitario en el siglo XXI. Revista redined, (56). Recuperado de http://www.um.es/ead/red/56/ prendes_et_al.pdf

Rangel, A. (2015). Competencias docentes digitales: propuesta de un perfil. Pixel-Bit. Revista de Medios y Educación, (46), 235-248. Recuperado de http://www.redalyc.org/articulo.oa?id=36832959015

Rivera-Vargas, P., Alonso-Cano, C., y Sancho-Gil, J. (2017). Desde la educación a distancia al e-Learning: emergencia, evolución y consolidación. Revista Educación y Tecnología, 1(10), 1-13. Recuperado de http://revistas.umce.cl/index.php/edytec/article/view/1127

Salas, I. y Umaña, A. C. (2017). Criterios de calidad para asignaturas en línea. En Consideraciones para el Diseño y Oferta de Asignaturas en Línea, pp. 130-136. Recuperado de https://www.uned.ac.cr/acade$\mathrm{mica} /$ images/vicerrectoria/documentacion/Consideraciones-diseno-oferta-asignaturas-linea.pdf

Salinas, J., De Benito, B. y Lizana, A. (2014). Competencias docentes para los nuevos escenarios de aprendizaje. Revista Interuniversitaria de Formación del Profesorado, 28(1), 145-163. Recuperado de http://www.redalyc.org/articulo.oa?id=27431190010 
Torra, I., De Corral, I., Pérez, M. J., Triadó, X., Pagès, T., Valderrama, E., Màrquez, M. D., Sabaté, S., Solà, P., Hernàndez, C., Sandrà, A., Guàrdia, L., Estebanell, M., Patiño, J., González, À., Fandos, M., Ruiz, N., Iglesias, M.C. y Tena, A. (2012). Identificación, desarrollo y evaluación de competencias docentes en la aplicación de planes de formación dirigidos a profesorado universitario. Red U: Revista de Docencia Universitaria (online), 10(2), 21-56. Recuperado de https://upcommons.upc.edu/bitstream/ handle/2117/18573/vol10_n2_REDU.pdf?sequence=1\&isAllowed=y

Tourón, J., Martín, D., Navarro Asencio, E., Pradas, S., e Iñigo, V. (2018). Validación de constructo de un instrumento para medir la competencia digital docente de los profesores (CDD). Revista Española de Pedagogía, 75(269), 25-54. https://doi.org/10.22550/REP76-1-2018-02

Turnitin (s. f.). Libro blanco. Integridad Académica en un mundo digital: Definición de plagio. Recuperado de https://www.uned.ac.cr/dpmd/pal/images/Part1_DefiningPlagiarism_Spanish.pdf

UNED (2017). Consideraciones para el Diseño y Oferta de Asignaturas en Línea. Recuperado de https:// www.uned.ac.cr/academica/images/vicerrectoria/documentacion/Consideraciones-diseno-ofertaasignaturas-linea.pdf

UNED (2018). Universidad Estatal a Distancia Informe de Labores 2018. Recuperado de https://www.uned. ac.cr/viplan/images/cppi/Informe_Labores_UNED_2018.pdf

UNED Asamblea Universitaria Representativa (2015). Lineamientos de Política Institucional 2015-2019. Recuperado de https://uned.ac.cr/academica/images/cidreb/Normativa/Lineamientos_politica_instituc_2015_2019.pdf

UNED Consejo Universitario (2019). Política para la Implementación del Diseño Universal para el Aprendizaje (DUA). Recuperado de https://www.uned.ac.cr/academica/images/ceced/docs/Acuerdo_Consejo_ Universitario.pdf

UNESCO (2019). Marco de competencias de los docentes en materia de TIC UNESCO. Recuperado de https:// unesdoc.unesco.org/ark:/48223/pf0000371024

Villarreal-Villa, S., García-Guliany, J., Hernández-Palma, H., y Steffens-Sanabria, E. (2019). Competencias Docentes y Transformaciones en la Educación en la Era Digital. Formación Universitaria, 12(6), 3-14. https://doi.org/10.4067/S0718-50062019000600003

Zabala, A. y Arnau, L. (2014). Métodos para la enseñanza de las competencias. Barcelona: Graó.

Zabalza, M. (2003). Competencias docentes del profesorado universitario. Calidad y desarrollo profesional. Madrid: Narcea. 